\title{
AC 2008-493: NAVAL ENGINEERING SUPPORT TEAM FOR THE AUVSI/ONR AUV COMPETITION
}

\section{Damien Bretall, NSWC}

Damien Bretall first began work at the Naval Surface Warfare Center as an intern in 2005. He has assisted with testing towed, underwater, and aircraft systems in the Marine and Aviation Department, including laser based experiments to evaluate the flow field around Navy vehicles and sensors. Recently, he completed his M.S. in Mechanical Engineering at the University of Maryland, focusing on Mechanics of Materials and the deformation of plates due to buried explosives.

\section{Deborah Furey, NSWC}




\title{
Naval Engineering Support Team for the AUVSI/ONR AUV Competition
}

\begin{abstract}
Robotics technology excites young people and fills them with ideas of possibility. Underwater robotics has an added element of difficulty and challenge that students accept and thrive on. Autonomous Unmanned Vehicle Systems International (AUVSI) is a foundation that has for decades encouraged the spread of unmanned vehicle technology both through professional conferences and student educational competitions. This group, together with the Office of Naval Research, has supported for the past 10 years a very successful international competition where student teams design, build and swim their own underwater robots called autonomous underwater vehicles (AUV). The competition involves designing and building an instrumented vehicle that will sense and respond to the underwater environment while completing an established set of challenges or tasks. In some cases, the biggest challenge is keeping the water on the outside of the vehicle!
\end{abstract}

Recently, with the support of ONR, there has been established a group of Navy engineers with diverse backgrounds to support and provide experience to the teams competing in the AUV competition. This ONR initiative intends to encourage the development of new engineers in order to instill the importance of naval engineering in the next generation. Representatives from around the United States work together to provide common guidance for new teams just joining the competition as well as detailed feedback on systems or hardware problems experienced by teams during the course of their vehicle development. Navy representatives include team members from the Naval Surface Warfare Center (NSWC) in Carderock, Maryland, NSWC Panama City, Florida, the Naval Undersea Warfare Center (NUWC) in Newport, Rhode Island, NUWC in Keyport, Washington, and Space and Naval Warfare (SPAWAR) Systems Center in San Diego, California. This group, called the Naval Engineering Support Team (NEST), has developed a collection of documents to help the teams with some of the vehicle technologies and competition logistics that could help them have a better competition experience. A primer document consisting of brief engineering reviews of relevant technologies such as hydrodynamics, propulsor systems, computers and underwater sensing has been completed and is available through the AUVSI website. Also, NEST is currently assembling a library of hardware for the teams to borrow during the year to assist them in developing their vehicle and to help them decide on types of components they might be interested in using in their platforms. NEST also conducts site visits to engage with the teams and provide additional interaction. One or two NEST members travel to individual schools or sites where the team has their vehicle to see their progress and provide information and feedback where possible. This paper presents the concept behind NEST and discusses the structure, operation and benefits that are hoped to be provided through this effort. 


\section{Introduction}

Autonomous robotics is an exciting up-and-coming field, and when combined with the added element of difficulty of operating underwater it presents a new set of challenges that students thrive on. It's hard enough for a robotic vehicle without having to worry about poor visibility, increased pressures, and the potential for flooding. Unlike some collegiate-level competitions, the objectives for the AUV competition have not been watered down (so to speak). It consists of tasks and obstacles challenging enough that, in 2007, only a select few teams completed the most difficult of them. However, it is apparent that students have enjoyed the challenge of designing and building an instrumented vehicle that will sense and respond to the underwater environment while completing an established set of challenges or tasks, possibly due to the cutting-edge nature of this objective.

The AUVSI and ONR International Autonomous Underwater Vehicle Competition is held every summer, with several dozen teams participating last year ${ }^{1}$. The tasks vary, but typically fall into one of several categories, as described in the NEST introductory presentation and illustrated in Figure $1^{2}$ :

- Basic Behaviors

○ Hold depth / heading / speed

- Navigate through a gate or hoop

- Move in response to sensor data

- Surface within a target region

- Acoustic Processing

$\circ$ Determine bearing to an acoustic source (range is helpful too)

○ Usually $\sim 20-30 \mathrm{kHz}$

- Vision Processing

- Identify lines or boxes and determine their position relative to the vehicle

$\circ$ Identify colors or flash rates of lights

- Perform a Task

○ Drop a marker or pick up an object at a specific location

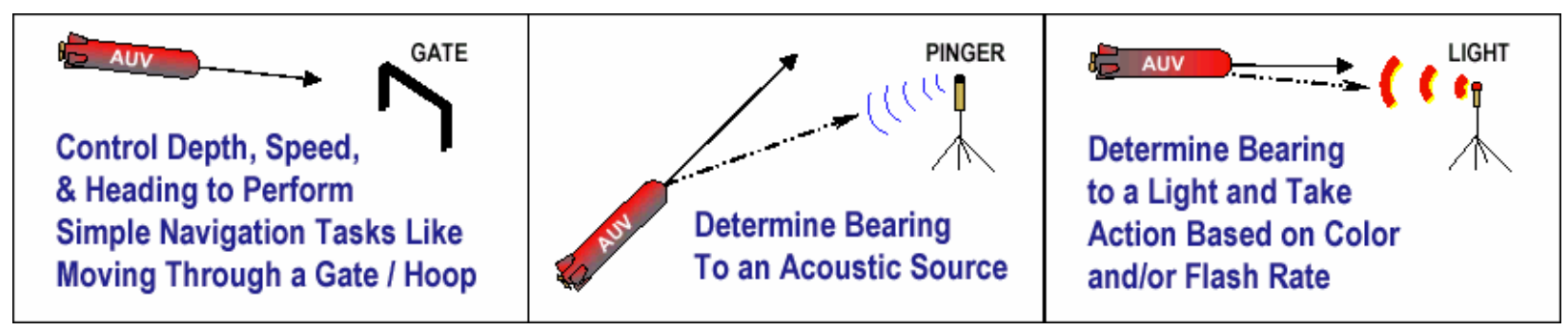

Figure 1: AUV Functional Requirements

As described in the NEST Primer document ${ }^{3}$, a vehicle that can successfully meet tasks such as these will need to be equipped with specific hardware designed to sense optical and acoustic signals. The vehicle will also need to be able to process this sensory input and respond appropriately. For this reason the AUV competition is a multidisciplinary challenge, and each team will need to integrate several different engineering areas into their final design. Examples 
of previous vehicles are shown in Figure 2. Some of the disciplines that are significant in developing such a platform are:

- Vehicle Hydrodynamics: ballasting, buoyancy, drag

- Underwater Propulsion: momentum, thrusters

- Underwater Sensing: visual, acoustic, heading

- Mechanical Systems: release mechanisms for the markers

- Power Systems: batteries for the propulsion system and onboard equipment, power distribution

- Heat Transfer: preventing the enclosed electronics from overheating

- Systems Engineering: system integration and testing

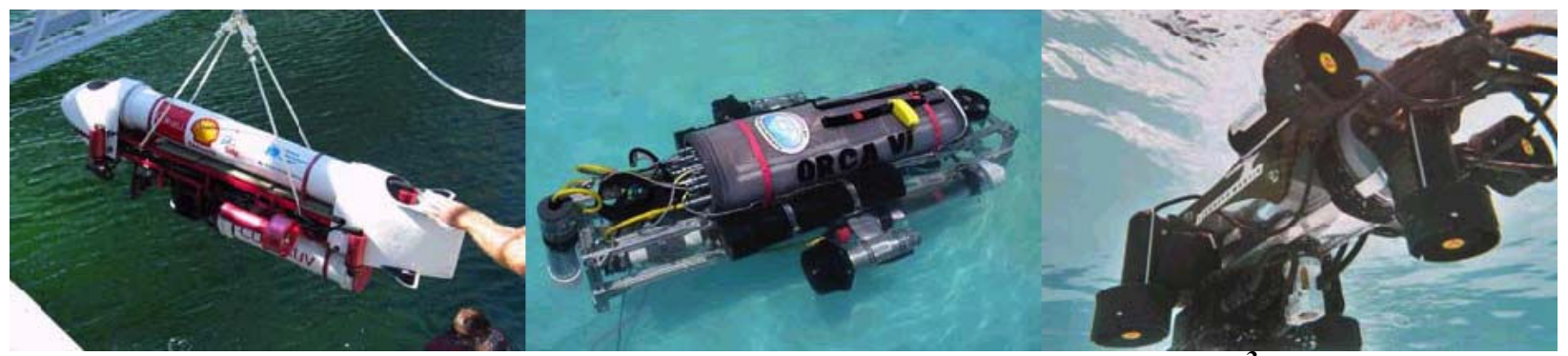

Figure 2: Examples of Competing Underwater Vehicles ${ }^{3}$

One of the unique aspects of this competition is that it encompasses teams from both the college and high school level, from a variety of engineering and scientific backgrounds. Teams of as small as two students and as large as several dozen have competed, and team size does not strongly correlate with success as can be the case with other competitions. The cost to create a competitive vehicle can also be quite reasonable. Lastly, the teams are closely supported by the Naval Engineering Support Team, an ONR initiative to encourage the development of new engineers in order to instill the importance of naval engineering in the next generation.

\section{Naval Engineering Support Team (NEST)}

Recently, with the support of ONR, there has been established a group of Navy engineers with diverse backgrounds to support and provide experience to the teams competing in the AUV competition. The NEST team is made up of Navy engineers and researchers from different Navy labs all around the United States. Navy representatives include team members from the Naval Surface Warfare Center (NSWC) in Carderock, Maryland, NSWC Panama City, Florida, the Naval Undersea Warfare Center (NUWC) in Newport, Rhode Island, NUWC in Keyport, Washington, and Space and Naval Warfare (SPAWAR) Systems Center in San Diego, California. The goal is to work with the teams on engineering, logistical and practical issues that come up throughout the year and to help each team be as best prepared as they possibly can be for the competition. NEST supports the teams throughout their design and testing process by helping with design questions, technical issues, team structure, fundraising techniques, issues about interfacing with sponsors, suggestions on testing the vehicle, and anything else within the NEST team's capabilities. This is achieved through a number of different means, such as visiting the students, communicating over email, message boards and conference calls, and distributing a document that covers almost every area that may be problematic for a student team. 


\section{$\underline{\text { Primer Document }}$}

NEST has also developed a collection of documents to help the teams with some of the vehicle technologies and competition logistics that could help them have a better experience. This document, referred to as the Primer ${ }^{3}$, consists of brief engineering reviews of relevant technologies such as hydrodynamics, propulsor systems, batteries, computers and underwater sensing. It was compiled with knowledge gained from previous competitors, judges, coordinators, and practicing engineers, and is available to all teams through the AUVSI website.

\section{$\underline{\text { Site Visits }}$}

One unique aspect of NEST is the diverse geographic locations of the members. This allows for site visits to student teams whenever the need arises, no matter which stage of development the team is in. It also provides for close interaction between the students and the engineers since there are only a handful of teams in each region. These benefits greatly outweigh the downside, which is that the meetings amongst NEST team must be held via teleconference. Face-to-face interaction is incredibly valuable to the student teams, whether they are just beginning and looking for some motivation, hammering out some design issues, conducting a mock technicalreview or testing their vehicle. For maximum benefit, the site visit is tailored to the experience level of the team; for brand new teams there is a general introductory presentation of the Primer material, and for more experienced teams there are design reviews and hardware demonstrations. This is discussed in more detail in the Recruiting and Team Development Stage section of this paper.

\section{$\underline{\text { Email and Message Boards }}$}

Another means for answering team's questions and concerns is through email communications and the AUVSI Underwater Competition forums. ${ }^{4}$ NEST members communicate with teams via email in order to answer technical questions, clarify rules, or simply monitor team progress. Students find it quite helpful that they are able to receive answers from experts in the field. Further, the AUVSI AUV forum provides an invaluable site for all teams to inquire and post concerns directly to the competition coordinators, as well as learn from some of the challenges other teams have encountered. NEST members also participate in the forum along with the students.

\section{$\underline{\text { Conference Calls }}$}

The NEST team regularly conducts conference calls in order to check the progress of the different regions. This includes sharing strategies and techniques used to reach out to schools, as well as discussing the progress of the students, which helps ensure that none of the schools fall too far behind. The group also discusses potential schools to reach out to, new information to include in the Primer documentation, as well as logistics for the competition. 


\section{NEST Support Throughout Various Stages}

NEST is able to help the teams with whatever is necessary through use of the above mentioned means. The role the NEST team plays varies greatly throughout the different stages leading up to the competition. It is unique to each student team's current situation, such as how many members they have returning from the previous year's competition.

\section{$\underline{\text { Recruiting and Team Development Stage }}$}

This stage refers to the initial period for teams that are either not yet well-established or have very few returning members. At this point they are often weighing the feasibility of participating in the AUV competition and beginning to form the basis of their team. One of the most important NEST goals for this stage is to provide the team with encouragement and motivation. The design and construction of a fully autonomous underwater vehicle can seem quite daunting at first, especially to a group of students who have never participated in a similar activity. However, admirable results have been obtained even by a team of two high school level students, proving that a modest budget and some hard work can go a long way. Stories such as this one, along with the reassurance that the NEST members will be a resource throughout the process, can provide the encouragement a young team needs. On top of this, the NEST members also motivate the teams by presenting a slide show with pictures from competitions, which helps show them that their hard work will be rewarded.

Another important NEST goal for this stage is to assist in team development. By recognizing potential shortcomings the NEST engineers can encourage the team to take corrective action. For example, although it is quite possible for a team of entirely electrical engineering majors to succeed, it would behoove them to seek out some more diverse members. The team could be improved by recruiting mechanical engineers to design the hull, finance and business majors to spearhead the fundraising, English majors to polish the technical documents and computer science majors to assist in the software programming and website development. Some tips for achieving this are also relayed, such as talking to the school to make the AUV competition count for class credit. This encourages more students to join, and allows them to devote more time to the project. At this stage it is also important to decide on a team organizational structure. Different possibilities are presented to the team, such as one focused on a systems approach and one focused on a more business approach, shown in Figure 3: 


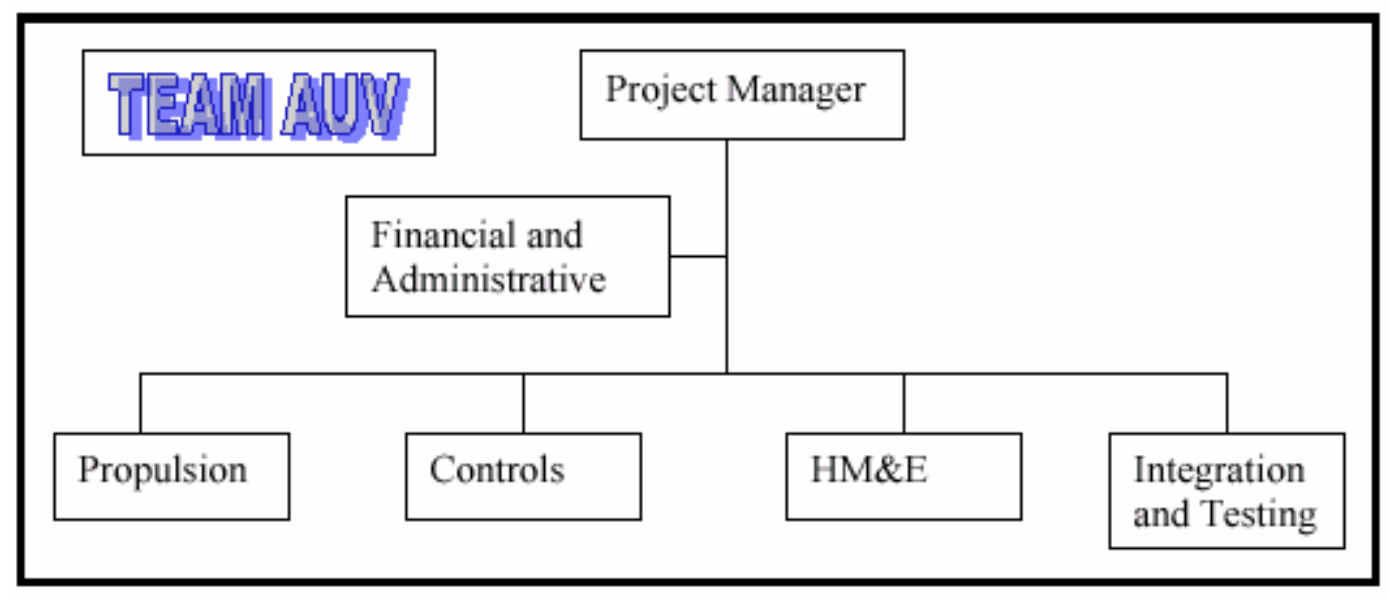

Team Organization Chart based on a systems approach.

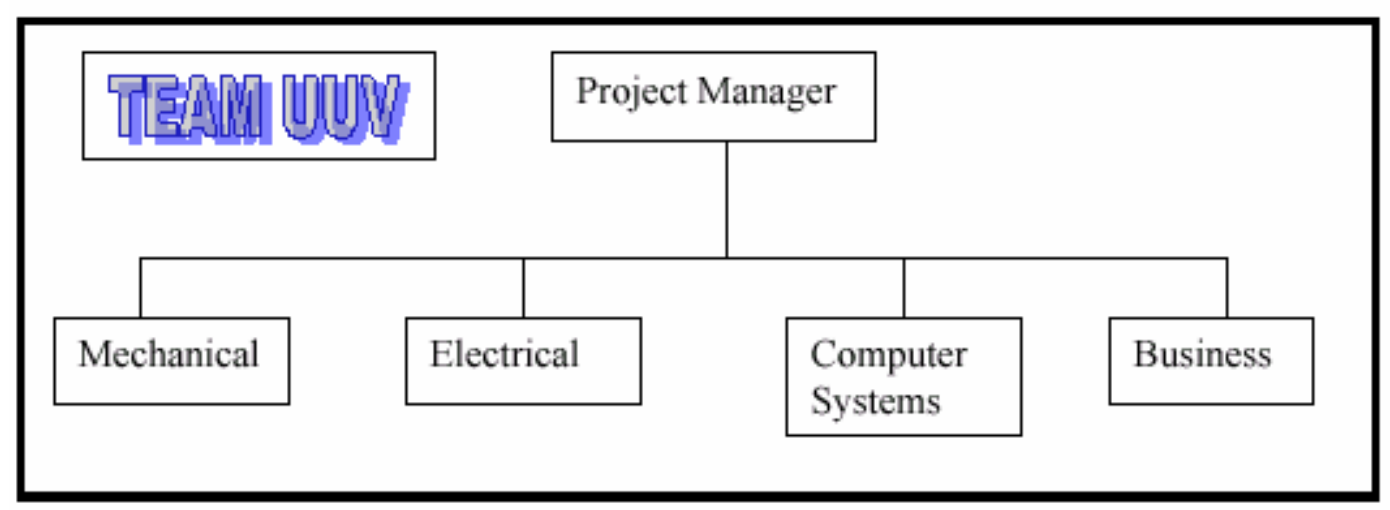

Team structure based on a business focus.

Figure 3: Different Possible Team Structures ${ }^{3}$

At this stage it is also important for the team to set appropriate goals and create a plan to achieve them. These goals must be uniquely tailored to the team and their current situation. For example, a first year team should set goals that are ambitious, yet still doable. If they expect to go in with a fresh slate and complete every single task they will probably become discouraged. A more realistic goal would be to first set out to achieve the simpler AUV tasks with the intention of developing a strong framework for future teams. This would make it more likely that the team will continue the next year and allow for the returning students to begin with a strong platform for success, rather than a fresh start. If the team being coached has competed in years past, it is important for them to recognize that they would benefit by successfully utilizing the efforts from the previous year. There is a lot to learn from past successes as well as mistakes. If the team has limited resources they can better focus them by avoiding the temptation to reinvent the wheel. The ultimate goal is to aid in focusing the teams toward the right direction and to facilitate a productive learning experience. 


\section{Design and Construction Stage}

The NEST members continue to support the teams as they move into the design stage. One of the goals is to help the teams avoid early mistakes and pitfalls that could prove to be costly later on. If resources become committed to a flawed design it is nearly impossible to salvage the spent capital and wasted time. This can easily be avoided during the design stage by a number of means. One simple solution is to connect the teams with nearby experts such as professors, or to refer them to NEST members with technical experience in the necessary areas. Another means of accomplishing this is by conducting an on-site technical review where the team presents their design plans to local NEST members, and potential improvements or flaws are discussed. At this stage it is also important to instill in the students the necessity to allow for the long lead times of certain critical parts. The NEST Primer document includes sections on just about any area the teams may be struggling with, or if they are looking for a comprehensive overview. The last NEST effort at this stage is to serve as a direct link to the AUVSI officials, especially in situations requiring rule clarification. This can help prevent wasted time and delays caused by waiting for a critical response, such as the legality of a chosen design feature.

Once construction has begun, it is not uncommon to run into new, unexpected challenges. The support team has the capabilities in providing technical assistance in any area that the team may need, such as water-proofing the electronics or programming the recognition software. This can be accomplished through an on-site visit or by connecting the team with an expert from one of the Navy labs around the United States.

\section{Testing and Competition Stage}

During the earlier stages it is emphasized to the teams that it is extremely helpful to conduct testing before the competition. If they are able to achieve this, the NEST engineers can help facilitate early testing and evaluation. It is also expected that interest in the AUV competition will eventually be high enough to hold several regional qualifying runs. This will give teams a chance to test their performance and improve before moving on to the international competition.

During the summer, NEST members from across the country travel to the AUVSI and ONR International Autonomous Underwater Vehicle Competition site at the Space and Naval Warfare Systems Center TRANSDEC Facility in San Diego, California. Here they can provide the competing teams with the assistance they need, whether they are simply putting on the finishing touches, or recovering from catastrophic occurrences, such as flooded electronics. The goal of the NEST members at this stage is to ensure all the students have a rewarding experience to show for all the efforts they have put in, and that all of the vehicles have a chance to exhibit their maximum potential. In fact, it is common at this point for the more successful teams to show a sense of camaraderie and lend a hand to the teams that need it the most.

\section{Next Steps}

Currently, NEST is developing a vehicle hardware library of items such as sensors, pingers, and compasses. This will allow teams to borrow and test out different hardware throughout the year, and enable them to make more informed purchasing decisions. They will be able to evaluate the 
potential onboard system before they invest their own funding. NEST is also actively pursuing new teams, and making sure potentially interested teams are given the guidance they need to avoid discouragement.

In the future, it is anticipated that there will be a great number of teams interested, making it possible to hold regional qualifying runs before the competition, and greatly increasing the exposure of autonomous unmanned vehicles. NEST will continue to encourage and assist the AUV teams throughout the year, and is currently developing a similar support structure for the new Autonomous Surface Vessel Competition (ASV).

\section{Acknowledgments}

NEST would like to thank Kelly Cooper from the Office of Naval Research for her continued support. We would also like to thank Daryl Davidson and Angela Carr from AUVSI, and Dave Novick from Sandia National Labs for all their input, support and enthusiasm that goes into the competition every year.

Additional acknowledgements also go out to the following members for supporting the NEST effort:

Benjamin Ruppel, Naval Surface Warfare Center Carderock Division

Dr. Kimberly Cipolla, Naval Undersea Warfare Center Newport Division

Ryan Stenson, Space and Naval Warfare Center San Diego

Andy Smith, Space and Naval Warfare Center San Diego

Julia Gazagnaire, Naval Surface Warfare Center Panama City

Dr. Signe Redfield, Naval Surface Warfare Center Panama City

Aamir Qaiyumi, Naval Surface Warfare Center Panama City

Jeremy Hatcher, Naval Surface Warfare Center Panama City

John Howard, Naval Undersea Warfare Center Keyport Division

\section{Bibliography}

1. AUVSI and ONR's 11th International Autonomous Underwater Vehicle Competition.

$<$ http://www.auvsi.org/competitions/water.cfm>

2. NEST Presentation. <http://www.auvsi.org/competitions/NEST.pdf $>$

3. NEST Primer Document. $<$ http://www.auvsi.org/competitions/PRIMER.pdf $>$

4. AUVSI Forum - Underwater. $<$ http://www.unsysinst.org/forum/viewforum.php?f=42> 